ARTICLE

DOI: $10.1038 /$ s41467-018-06774-1

\title{
TRIP13 and APC15 drive mitotic exit by turnover of interphase- and unattached kinetochore-produced MCC
}

Dong Hyun Kim,2, Joo Seok Han ${ }^{3}$, Peter Ly (1) 1,2, Qiaozhen Ye², Moira A. McMahon 1,2,6, Kyungjae Myung 3,4, Kevin D. Corbett (1) ${ }^{2,5} \&$ Don W. Cleveland ${ }^{1,2}$

The mitotic checkpoint ensures accurate chromosome segregation through assembly of the mitotic checkpoint complex (MCC), a soluble inhibitor of the anaphase-promoting complex/ cyclosome (APC/C) produced by unattached kinetochores. MCC is also assembled during interphase by Mad1/Mad2 bound at nuclear pores, thereby preventing premature mitotic exit prior to kinetochore maturation and checkpoint activation. Using degron tagging to rapidly deplete the AAA + ATPase TRIP13, we show that its catalytic activity is required to maintain a pool of open-state Mad2 for MCC assembly, thereby supporting mitotic checkpoint activation, but is also required for timely mitotic exit through catalytic disassembly of MCC. Strikingly, combining TRIP13 depletion with elimination of APC15-dependent Cdc20 ubiquitination/degradation results in a complete inability to exit mitosis, even when MCC assembly at unattached kinetochores is prevented. Thus, mitotic exit requires MCC produced either in interphase or mitosis to be disassembled by TRIP13-catalyzed removal of Mad2 or APC15-driven ubiquitination/degradation of its Cdc20 subunit.

\footnotetext{
${ }^{1}$ Ludwig Institute for Cancer Research, San Diego Branch, La Jolla, CA 92093, USA. ${ }^{2}$ Department of Cellular and Molecular Medicine, University of California-San Diego, La Jolla, CA 92093, USA. ${ }^{3}$ Center for Genomic Integrity, Institute for Basic Science (IBS), Ulsan 44919, Republic of Korea. ${ }^{4}$ School of Life Sciences, Ulsan National Institute for Science and Technology (UNIST), Ulsan 44919, Republic of Korea. ${ }^{5}$ Department of Chemistry, University of California-San Diego, La Jolla, CA 92093, USA. ${ }^{6}$ Present address: Ionis Pharmaceuticals, 2855 Gazelle Ct, Carlsbad, CA 92010, USA. Correspondence and requests for materials should be addressed to K.D.C. (email: kcorbett@ucsd.edu) or to D.W.C. (email: dcleveland@ucsd.edu)
} 
$\mathrm{T}$ he mitotic checkpoint (or spindle-assembly checkpoint) delays the onset of anaphase until all chromosomes have successfully attached to spindle microtubules, thereby serving as the primary guard against chromosome missegregation. Anaphase onset is controlled by a multisubunit E3 ubiquitin ligase, the anaphase-promoting complex/cyclosome $(\mathrm{APC} / \mathrm{C})^{1-4}$. The APC/C, together with its co-activator Cdc20, targets cyclin B and securin for proteasome-mediated destruction, thereby causing exit from mitosis and activation of separase [reviewed in ref. ${ }^{5}$. The mitotic checkpoint functions through unattached kinetochores, which generate a soluble mitotic checkpoint complex (MCC) consisting of Mad2, Cdc20, BubR1, and Bub3. The MCC binds the APC/C ${ }^{C d c 20}$ complex as a substrate analog and directly inhibits its activity ${ }^{6-8}$. While MCC is mainly produced in mitosis by the mitotic checkpoint, low levels of MCC are also produced in interphase cells, and this pool of MCC is thought to be important for inhibiting early mitotic exit prior to kinetochore maturation and mitotic checkpoint activation ${ }^{9-12}$.

The key component controlling MCC assembly is Mad2, an essential checkpoint protein containing a HORMA domain ${ }^{13}$ that can adopt two different conformations: "open" (O-Mad2) or "closed" (C-Mad2). C-Mad2 binds partner proteins, including Cdc20 and Mad1, through a distinctive "seat belt" interaction, in which the Mad2 C-terminus embraces a short Mad2-interacting motif (MIM) in the partner. In O-Mad2, the C-terminal seat belt occupies the MIM binding site to prevent complex formation $^{14-17}$. In vivo and in vitro evidence has shown that O-Mad2 is recruited to Mad1:C-Mad2 complexes at unattached kinetochores, where it is converted to C-Mad2 and assembled with Cdc20 to form the core of the mitotic checkpoint complex ${ }^{18-22}$. After assembly of the Cdc20:C-Mad2 complex, recruitment of BubR1 and Bub3 complete assembly of MCC, which binds to $\mathrm{APC} / \mathrm{C}^{\mathrm{Cdc} 20}$ and inhibits its recognition of cyclin B and securin, thereby delaying mitotic exit. Low-level MCC assembly in interphase is thought to occur through a similar mechanism, catalyzed by Mad1:C-Mad2 localized to nuclear pores ${ }^{11,23}$.

Once all kinetochores have become attached to microtubules, the mitotic checkpoint is silenced and $\mathrm{APC} / \mathrm{C}^{\mathrm{Cdc} 20}$ is reactivated for cyclin $\mathrm{B}$ and securin ubiquitination and their subsequent proteasomal destruction, which in turn initiates anaphase onset. At each kinetochore, stable microtubule attachment releases the Mad1:CMad2 complex ${ }^{24-27}$ and PP1 phosphatase activity is elevated to inactivate checkpoint kinases, including Mps1, thereby leading to suppression of additional MCC production ${ }^{28-33}$. Existing $\mathrm{APC} / \mathrm{C}^{\mathrm{Cdc} 20}$-bound MCC (APC/C $\mathrm{C}^{\mathrm{Cdc} 20-\mathrm{MCC}}$ ) must then be disassembled and/or degraded to reactivate $\mathrm{APC} / \mathrm{C}^{\mathrm{Cdc} 20}$ recognition of cyclin B and securin. One proposed mechanism for $\mathrm{APC} / \mathrm{C}^{\mathrm{Cdc} 20}$ reactivation is ubiquitination of the Cdc20 subunit of MCC by the inhibited $\mathrm{APC} / \mathrm{C}^{\mathrm{Cdc} 20}$ itself, which is enabled by a conformational change within the $\mathrm{APC} / \mathrm{C}$ allowing recruitment of an $\mathrm{E} 2$ enzyme and ubiquitination of Cdc20 in MCC $34-37$. Loss of APC15, a $14 \mathrm{kD}$ $\mathrm{APC} / \mathrm{C}$ subunit which is dispensable for APC/C assembly and activity ${ }^{38-40}$, eliminates this reactivation pathway by preventing the conformational change required for E2 recruitment and Cdc20 ubiquitination ${ }^{41,42}$. A second pathway potentially driving mitotic exit has been proposed to involve the AAA + ATPase TRIP13 acting with an adapter protein $\mathrm{p} 31^{\text {comet }}$ to extract Mad2 from the assembled MCC. p31 ${ }^{\text {comet }}$ specifically binds C-Mad2 alone or in complex with Cdc20 and other MCC subunits ${ }^{43-46}$. TRIP13 and p31 comet together can mediate disassembly of MCC complexes in vitro ${ }^{47-49}$, acting by partially unfolding the $\mathrm{N}$-terminus of CMad2 to catalyze its conversion to O-Mad2 $2^{50-52}$. Whether and how the two proposed pathways for MCC disassembly/degradationAPC15-mediated ubiquitination/degradation and TRIP13-mediated disassembly - synergize to mediate $\mathrm{APC} / \mathrm{C}^{\mathrm{Cdc} 20}$ activation and mitotic checkpoint silencing is not known.
Despite its proposed role in MCC disassembly and checkpoint silencing, deletion or complete loss of TRIP13 in C. elegans ${ }^{53}$ or human cells ${ }^{54-56}$ causes defects in checkpoint activation, rather than failure of checkpoint silencing. In the absence of TRIP13, most soluble Mad2 is in the closed conformation, rendering it unable to be recruited to unattached kinetochores and assembled with $\mathrm{Cdc} 20$ into the $\mathrm{MCC}^{54}$. This finding, plus the observation that purified O-Mad2 spontaneously converts to C-Mad2 in vitro over the course of several hours ${ }^{17}$, suggests that TRIP13 is required for mitotic checkpoint activation by maintaining a pool of O-Mad2.

The proposed dual roles of TRIP13 in the mitotic checkpoint mirror a similar duality in its observed roles in cancer. Recently, it was shown that biallelic loss-of-function mutations in TRIP13 underlie mosaic variegated aneuploidy and Wilms tumor in children, establishing TRIP13 as a bona fide tumor suppressor ${ }^{55}$. Conversely, several studies have highlighted that TRIP13 is overexpressed in many cancer types, and that high-level TRIP13 expression correlates with poor patient outcomes ${ }^{57,58}$. The contributions of TRIP13 to tumorigenesis and cancer progression remain largely mysterious.

Here, we develop an inducible system to rapidly and completely deplete TRIP13 in cultured human cells by engineering auxin-inducible degron (AID) tags into both endogenous TRIP13 loci. Loss of TRIP13 during interphase is shown to trigger checkpoint activation failure in the next mitosis due to a loss of soluble O-Mad2. Conversely, cells that enter mitosis soon after depletion of TRIP13, prior to full O-Mad2 to C-Mad2 conversion, show a dramatic checkpoint-mediated delay in mitotic exit, revealing TRIP13's critical role in checkpoint silencing. Further, we show that checkpoint silencing involves TRIP13 acting synergistically with APC15-mediated Cdc20 ubiquitination to disassemble or degrade, respectively, soluble and APC/C $\mathrm{C}^{\mathrm{Cd} 20}$-bound MCC. Strikingly, we find that mitotic exit requires MCC produced either in interphase or mitosis to be disassembled by TRIP13-catalyzed removal of Mad2 or APC15-driven ubiquitination/degradation of its Cdc20 subunit.

\section{Results}

Long-term TRIP13 depletion compromises cell fitness. To determine the roles of TRIP13 in the mitotic checkpoint, we sought to develop a means of acutely controlling TRIP13 levels in live cells. We used CRISPR/Cas9 to sequentially introduce sequences encoding green fluorescent protein (GFP) and an auxin-inducible degron (AID) $)^{59,60}$ into both TRIP13 alleles of nearly diploid human DLD-1 cells (hereafter, TRIP13 ${ }^{\text {AID }}$ ) also stably expressing a fluorescent histone $\mathrm{H} 2 \mathrm{~B}$ protein $(\mathrm{H} 2 \mathrm{~B}-$ mRFP) and the TIR1-9Myc E3 ubiquitin ligase to permit inducible ubiquitination of AID-tagged proteins (Fig. 1a). Upon addition of indole-3-acetic acid (IAA), GFP-AID-TRIP13 was rapidly degraded with a $t_{1 / 2}$ of $\sim 7 \mathrm{~min}$ (Fig. 1b, Supplementary Fig. 1A), becoming undetectable (to $<3 \%$ of its initial level) within $30 \mathrm{~min}$. No discernable difference in growth between normal or TRIP13-depleted cells was seen after 3 days (Supplementary Fig. 1B). We first used time-lapse live-cell imaging (using H2B-mRFP to monitor chromosome condensation and decondensation) to examine the fate of cells in the first mitosis after TRIP13 depletion. Addition of nocodazole to inhibit spindle microtubule assembly caused a chronic mitotic arrest in TRIP13-containing cells (Fig. 1c, left panel), whereas most cells entering mitosis $\geq 24 \mathrm{~h}$ after TRIP13 depletion exited mitosis within $40 \mathrm{~min}$ (Fig. 1c; right panel). In unperturbed mitoses (no nocodazole), the time from nuclear envelope breakdown (NEBD) to chromosome alignment in metaphase 
was indistinguishable in the presence or absence of TRIP13 ( 23 min, Fig. 1d; left panel), but in the absence of TRIP13 the transition from metaphase-to-anaphase was accelerated (lowering it from $\sim 20$ to $\sim 11 \mathrm{~min}$; Fig. 1d, right panel). Normal metaphase-to-anaphase timing could be rescued in TRIP13depleted cells by expression of wild-type TRIP13, but not with TRIP13 bearing the Walker B motif E253Q mutation (TRIP13 ${ }^{\mathrm{EQ}}$ ), which allows for ATP binding and stabilizes the TRIP13 hexamer, but eliminates ATP hydrolysis ${ }^{50,52}$ (Supplementary Fig. 1C-F). These findings demonstrate that TRIP13 is not strictly needed for entry into mitosis or for chromosome congression, but is required for activation and/or maintenance of the mitotic checkpoint.

Live-cell imaging of TRIP13 $3^{\text {AID }}$ cells revealed significant errors in chromosome segregation during anaphase of the first mitosis after TRIP13 depletion (Supplementary Fig. 1G) that drove chromosomal aneuploidy in the daughter cells (as determined by fluorescence in situ hybridization (FISH) analyses-Fig. 1e). Clonogenic assays indicated that prolonged (3 week) depletion of TRIP13 resulted in significantly smaller colony size compared to TRIP13WT cells (Supplementary Fig. 1H). Collectively, this evidence demonstrates that TRIP13 normally acts to prevent aneuploidy and that loss of TRIP13 compromises long-term cell fitness.

TRIP13 maintains open Mad2 to support checkpoint activation. To examine Mad2 conformation in the absence of TRIP13, we depleted TRIP13 in TRIP13 ${ }^{\text {AID }}$ cells by addition of IAA, and examined Mad2 conformation in cell lysates by Mono-Q ionexchange chromatography (which separates the two Mad2

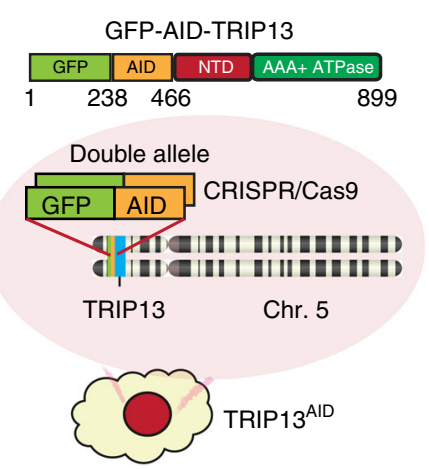

DLD-1 T-REx FIp-In H2B ${ }^{\text {mRFP }}$ TIR1-9Myc b

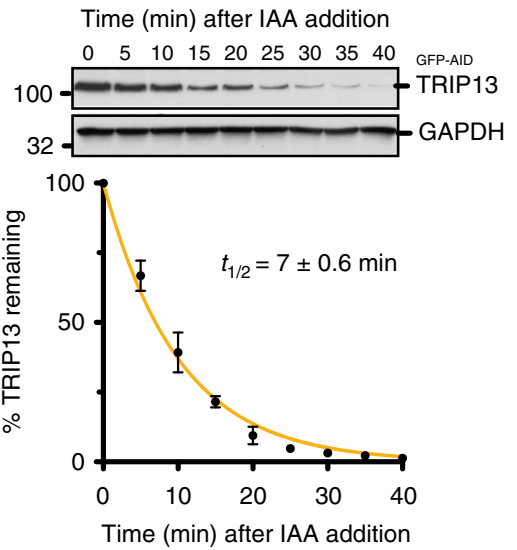

C Degrade TRIP13
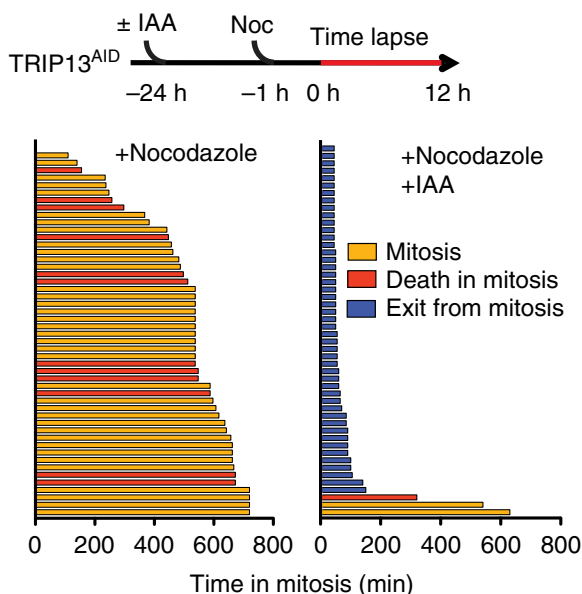

d

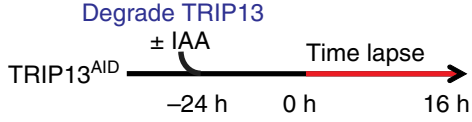

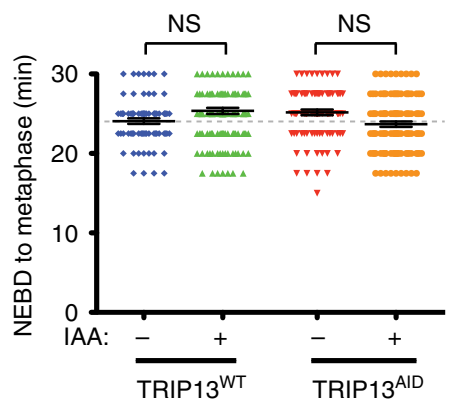

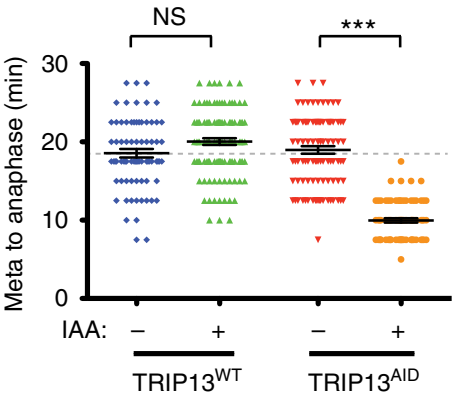

e

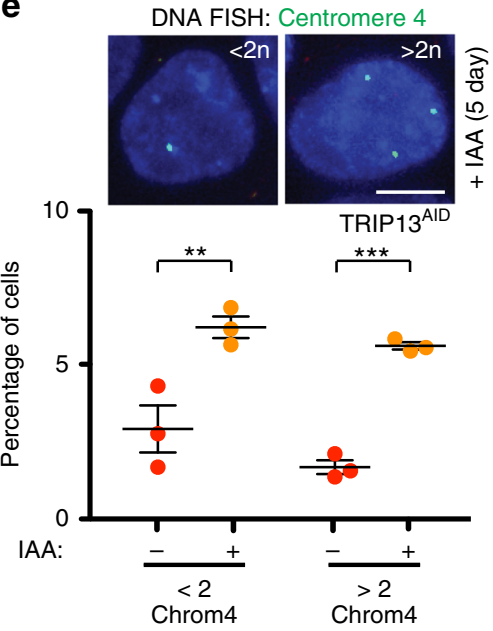

Fig. 1 Abrupt TRIP13 depletion compromises mitotic checkpoint activation. a Schematic illustration of CRISPR-Cas9 mediated genome editing of DLD-1 TREx Flp-In H2B mRFP + TIR1-9Myc ${ }^{+}$human colon cancer cell line to tag both endogenous TRIP13 alleles with green fluorescent protein (GFP) and an auxininducible degron (AID). b Upper: Indole-3-acetic acid (IAA) was added to engineered TRIP13AID cells and level of GFP-AID-TRIP13 was measured by quantitative immunoblot analysis. Lower: Degradation kinetics of GFP-AID-TRIP13 from triplicate measurements, fit using a single-exponential decay function (yellow line). c TRIP13 function in mitotic checkpoint. Upper: Schematic of experiment to test the role of TRIP13 in mitotic checkpoint activation. Lower: Timing of mitotic exit and cell fate as measured by time-lapse live-cell imaging. ( $n=57$ for +Nocodazole, $n=54$ for + Nocodazole, + IAA). $\mathbf{d}$ TRIP13 function in unperturbed mitosis. Upper: Schematic of experiment to test the contribution of TRIP13 in mitotic checkpoint activation. Lower: For each cell, timing of nuclear envelope breakdown (NEBD) to metaphase (left panel) and metaphase-to-anaphase (right panel) were measured. Cells monitored: $n=$ $78,137,100,120,79,129,102,81$. e Upper: Representative images of TRIP13AID cells treated with IAA for 5 days and probed by FISH for the centromere of chromosome 4. Lower: Percentage of cells with the indicated number of chromosome 4 centromere foci after 5-day IAA treatment. Total cells counted: 1156 for control, 1119 for IAA treatment over three separate experiments. Scale bar, $5 \mu \mathrm{m}$. $P$-values for $\mathbf{d}$ and $\mathbf{e}$ were calculated using an unpaired two-tailed t-test $\left({ }^{\star \star} P<0.01,{ }^{\star \star \star} P<0.001\right.$, NS not significant). Lines in $\mathbf{b}, \mathbf{d}$, and e represent the mean \pm s.e.m. 
a

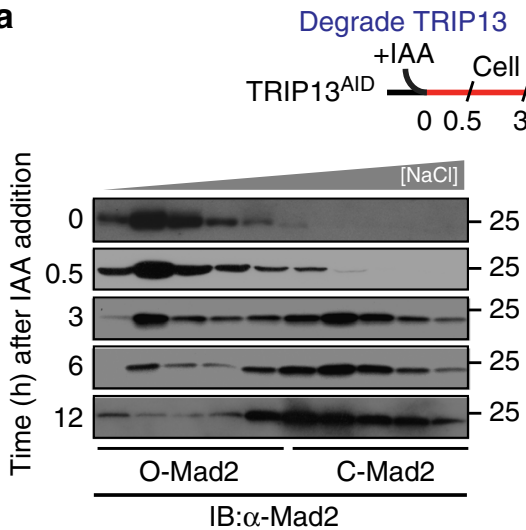

C
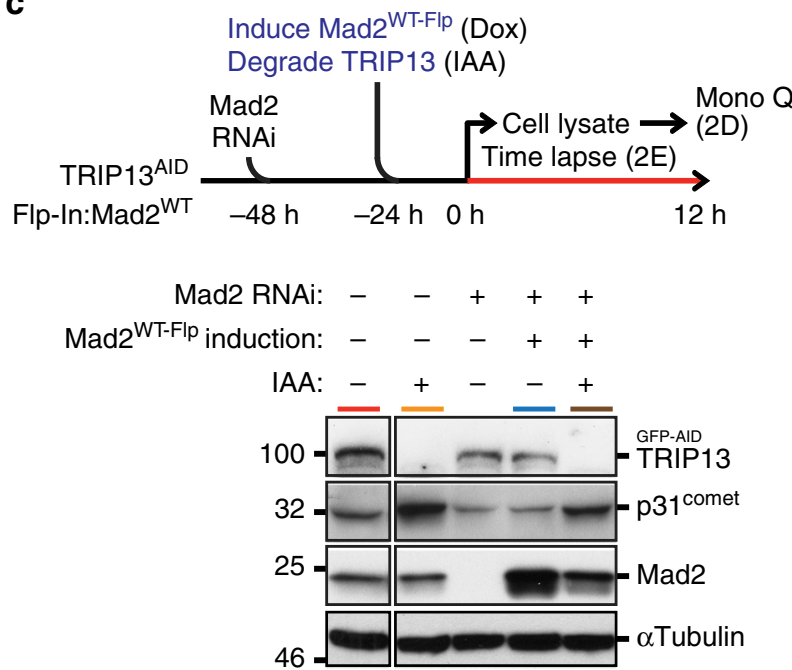

f Degrade TRIP13

+IAA FACS: Phospho-H3(Ser10)
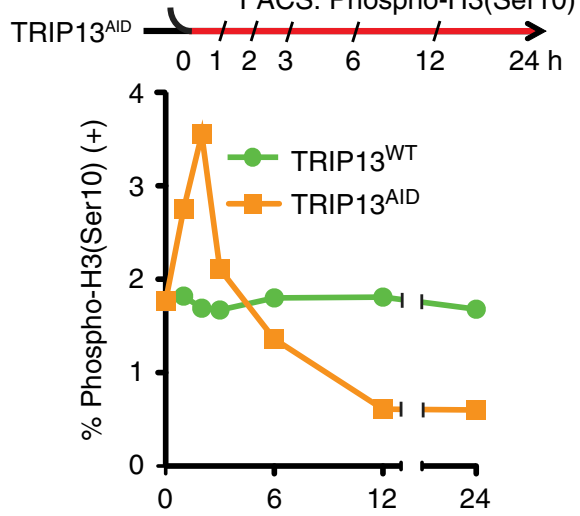

Time (h) after IAA treatment

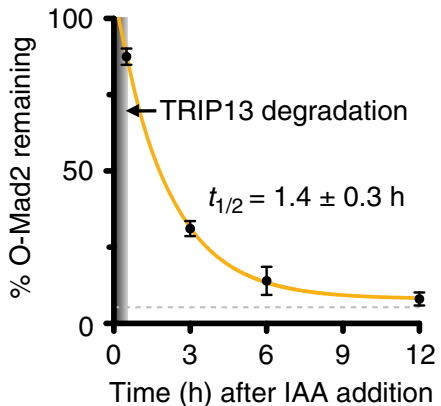

b
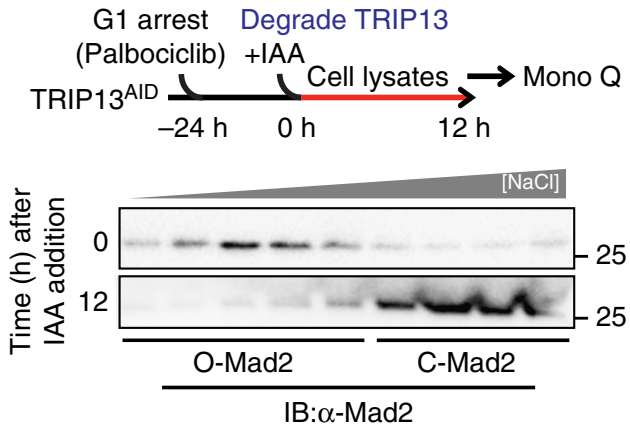

d

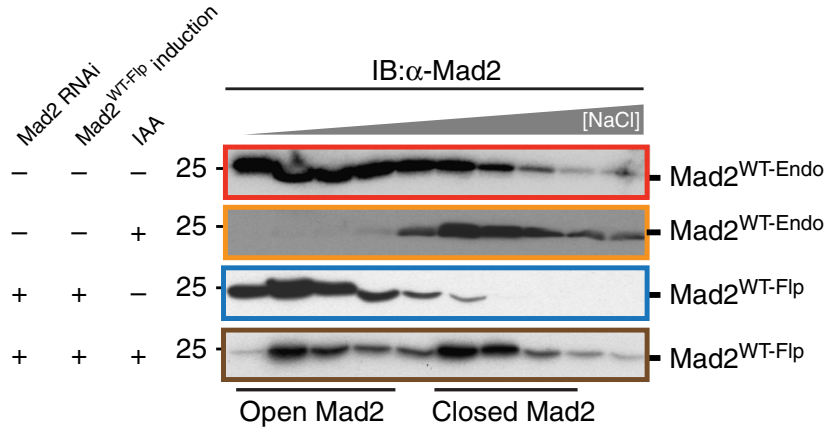

e

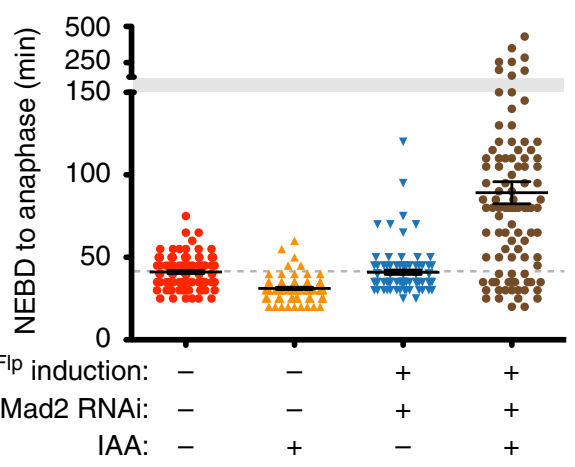

Degrade TRIP13
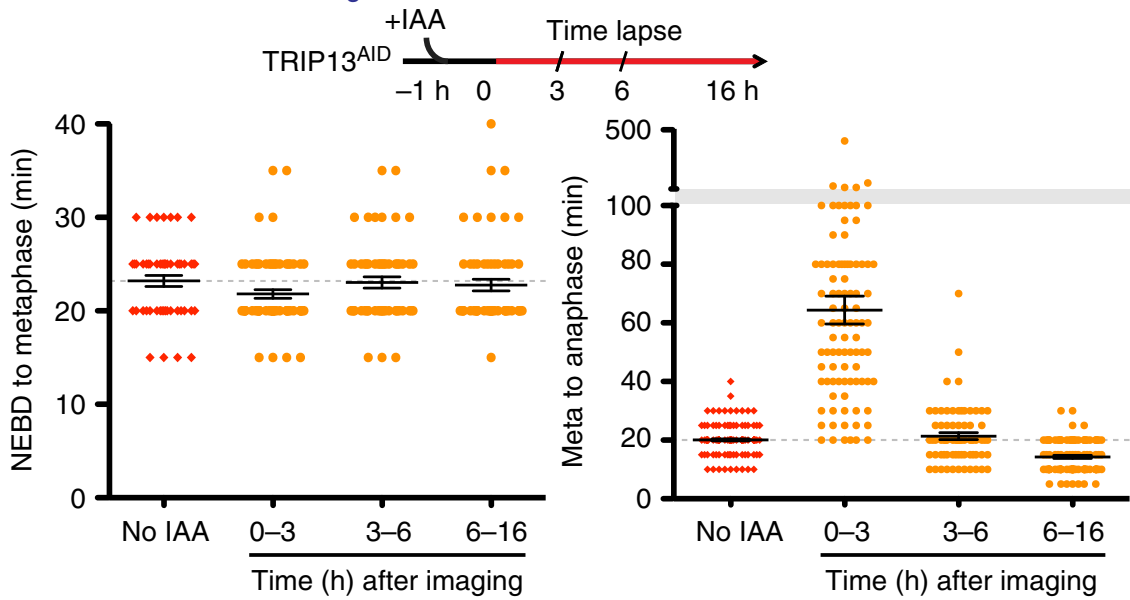

conformers $16,17,50,52)$. Mad2 was mostly in its open conformer prior to TRIP13 depletion, in agreement with prior studies ${ }^{17}$ (Fig. 2a). Over time, however, Mad2 gradually converted to the closed conformation, with a conversion half-time of $\sim 1.4 \mathrm{~h}$ and reaching an equilibrium at $\sim 8 \% \mathrm{O}-\mathrm{Mad} 2$ (Fig. $2 \mathrm{a}$, right panel).
This rate of conformational conversion is consistent with the in vitro rate of spontaneous $\mathrm{O}-\mathrm{Mad} 2$ to $\mathrm{C}-\mathrm{Mad} 2$ conversion (half-time; $1.5-2 \mathrm{~h})^{61}$ and with the equilibrium percentage of $\mathrm{O}$ Mad2 seen in our earlier in vitro measurements ${ }^{50}$. These data clearly indicate that a major role of TRIP13 is to counteract 
Fig. 2 TRIP13 regulates mitotic checkpoint activation and silencing through Mad2. a Upper: Schematic of experiment to test kinetics of Mad2 conformational conversion after TRIP13 depletion. Lower left: Mono-Q ion-exchange chromatography analysis of soluble Mad2 in TRIP13AID cells after IAA treatment for the indicated times. Lower right: Kinetics of O-Mad2 to C-Mad2 conversion was calculated from triplicate measurements, fit using a singleexponential decay function (yellow line). $t_{1 / 2}=1.4 \pm 0.3 \mathrm{~h}$, plateau $=8 \%$ O-Mad2 (gray dashed line). b Mad2 conformation in G1 after TRIP13 depletion. TRIP13AID cells were arrested in G1 with Palbociclib and Mad2 conformation was measured by Mono-Q ion-exchange chromatography at 0 or $12 \mathrm{~h}$ after addition of IAA to degrade TRIP13. c Upper: Schematic of the approach to replace endogenous Mad2 in TRIP13-depleted cells (used in d and e). Lower: Immunoblot analysis of the replacement of endogenous Mad2 with Mad2WT-Flp induced by doxycycline. $\mathbf{d}$ Mono-Q ion-exchange chromatography analysis of Mad2 conformation after replacement of endogenous Mad2 with Mad2WT-Flp, in the presence (blue box) or absence (brown box) of TRIP13. High-level expression of Mad2WT-Flp resulted in a significant O-Mad2 population even after TRIP13 depletion. e Mitotic timing (NEBD-to-anaphase) after replacement of endogenous Mad2 with Mad2WT-Flp, measured by time-lapse live-cell imaging. ( $n=100$ for each condition). $\mathbf{f}$ Upper: Schematic of experiment to measure population of mitotic TRIP13WT and TRIP13AID cells upon IAA treatment. Lower: Percentage of phospho-histone H3 (Ser10) positive cells was measured using flow cytometry at different time points after IAA treatment. Detailed FACS profiles in Supplementary Fig. 2D. g Upper: Schematic of experiment to measure mitotic timing in TRIP13AID cells upon IAA treatment. Lower: NEBD-to-metaphase (left) and metaphase-to-anaphase (right) timing of TRIP13AID cells at different time points after IAA addition. (From left to right panel, $n=50,69,56,58,176,93,70$, and 110). Lines in $\mathbf{e}$ and $\mathbf{g}$ represent the mean \pm s.e.m.

spontaneous conversion of O-Mad2 to C-Mad2, the latter of which cannot be recruited to unattached kinetochores and assembled into the MCC. Importantly, this conversion was not related to cell-cycle progression: in cells arrested in G1 (by addition of the Cdk4/6 inhibitor palbociclib ${ }^{62}$ ), $>90 \%$ of $\mathrm{Mad} 2$ converted to the closed conformation within $12 \mathrm{~h}$ of TRIP13 depletion (Fig. 2b).

Cells chronically depleted of TRIP13 have been reported to show changes in overall Mad2 and $\mathrm{p} 31^{\text {comet }}$ protein levels compared to normal cells ${ }^{54,55}$. We observed similar changes upon TRIP13 depletion, including a $\sim 2$-fold reduction in Mad2 level and a $>5$-fold increase in p31 ${ }^{\text {comet }}$ levels (Supplementary Fig. 3). When we replaced endogenous $\mathrm{Mad} 2$ with a mutant $(\operatorname{Mad} 2 \mathrm{H} 191 \mathrm{~A})$ that predominantly adopts the open conformation even in the absence of TRIP $13^{52,63}$, we did not observe increased p $31^{\text {comet }}$ levels upon TRIP13 depletion (Supplementary Fig. 3F). Thus, p31 $1^{\text {comet }}$ is stabilized by binding C-Mad2 and strongly accumulates in the absence of TRIP $13^{54}$.

To test whether the checkpoint activation defect upon longerterm TRIP13 depletion was caused by insufficient O-Mad2, we stably integrated a gene encoding untagged, wild-type Mad2 into a doxycycline-inducible FRT locus in the TRIP13 ${ }^{\text {AID }}$ cell line (hereafter Mad2WT-Flp). We then depleted endogenous Mad2 by siRNA and induced the Mad2WT-Flp gene (whose mRNA is resistant to the siRNA) (Fig. 2c). In the presence of TRIP13, most Mad2 produced from either the endogenous or Mad2WT-Flp genes was in the form of O-Mad2 (Fig. 2d) and the cells traversed mitosis with normal timing (Fig. 2e). In contrast to the nearly complete conversion of endogenous Mad2 (Mad2WT-endo) to the closed form after TRIP13 degradation (Fig. 2d), doxycyclinemediated synthesis of new Mad2 resulted in a significant pool of soluble O-Mad2 even in the absence of TRIP13 (Fig. 2d), which in turn supported a strong delay in the timing of mitotic exit in these cells (Fig. 2e). Thus, newly synthesized Mad2 adopts the $\mathrm{O}-\mathrm{Mad} 2$ conformation and this new Mad2 is sufficient to rescue mitotic checkpoint activation in the absence of TRIP13, while the subsequent delay in mitotic exit supports a role for TRIP13 in MCC disassembly to permit anaphase onset.

TRIP13 has a critical role in mitotic checkpoint silencing. In vitro, TRIP13 and its Mad2-binding adapter protein p31 comet have been shown to convert C-Mad2 to $\mathrm{O}-\mathrm{Mad} 2^{50}$ and to release Mad2 from Cdc20:C-Mad2 or the four-protein MCC $47,48,64$. These findings, plus reports that RNAi-mediated reduction of TRIP13 can slow mitotic exit in some cells ${ }^{49}$, have led to the idea that TRIP13 and p31 $1^{\text {comet }}$ participate in checkpoint silencing. We initially tested a role for TRIP13 in checkpoint silencing by measuring the mitotic index (by scoring histone $\mathrm{H} 3$ serine 10 phosphorylation-positive cells) over time after inducing TRIP13 degradation. This revealed a sharp, transient increase in mitotic cells between 1 and $3 \mathrm{~h}$ after TRIP13 degradation, consistent with a TRIP13 role in mitotic checkpoint silencing and timely mitotic exit (Fig. 2f). We then used time-lapse live-cell imaging to determine that, despite unaffected NEBD-to-metaphase timing, metaphase-to-anaphase timing was significantly delayed (more than tripling the typical 20 to $64 \mathrm{~min}$ ) in cells entering mitosis between 0 and $3 \mathrm{~h}$ after TRIP13 degradation (Fig. 2g). Cells entering mitosis 3-6h after TRIP13 degradation exited mitosis with nearly wild-type timing, while cells entering mitosis even later (6-16 h after TRIP13 degradation) exited mitosis faster than wild-type cells (Fig. $2 \mathrm{~g}$ ), consistent with failure of mitotic checkpoint activation and with the mitotic timing we observed after 24-h TRIP13 depletion (Fig. 1d) and in TRIP13 ${ }^{-1-}$ cells $^{54}$.

To more clearly establish that the mitotic exit delay we observed shortly after TRIP13 depletion was due to a defect in mitotic checkpoint silencing, we next tested whether this delay depended on Mad2 and actively signaling kinetochores. We used siRNA to deplete Mad2 in TRIP13 ${ }^{\text {AID }}$ cells and then examined mitotic timing shortly after TRIP13 depletion. Mad2-depleted cells exited mitosis significantly faster than untreated cells regardless of whether or not TRIP13 was present (Supplementary Fig. 2A). We next prevented MCC assembly at unattached kinetochores by addition of reversine to inactivate the Mps1 kinase ${ }^{65}$, and found that this treatment also accelerated mitotic exit both in the presence and absence of TRIP13 (Supplementary Fig. 2B). Together, these data indicate that the delay in mitotic exit that we observe shortly after TRIP13 depletion depends on both Mad2 and actively signaling kinetochores, supporting a direct role for TRIP13 in checkpoint silencing, most likely through MCC disassembly after kinetochore-microtubule attachment.

TRIP13 and APC15 act in parallel to inactivate MCC. Despite the strong delay in mitotic exit in cells that entered mitosis within $3 \mathrm{~h}$ of TRIP13 degradation, these cells did eventually exit mitosis. This observation indicated that MCC bound to $\mathrm{APC} / \mathrm{C}^{\mathrm{Cdc} 20}$ is either able to spontaneously disassemble, or that it is disassembled/degraded through an alternate pathway(s). Beyond TRIP13-mediated disassembly of Mad2 from MCC ${ }^{48,49}$, another proposed pathway involves ubiquitination of Cdc20 within MCC bound to $\mathrm{APC} / \mathrm{C}^{\mathrm{Cdc} 20}$, resulting in $\mathrm{Cdc} 20$ degradation, MCC disassembly, and reactivation of $\mathrm{APC} / \mathrm{C}^{\mathrm{Cdc} 20}$ for recognition of cyclin B and securin ${ }^{34-37}$. For this second pathway, APC15, a 14 
a

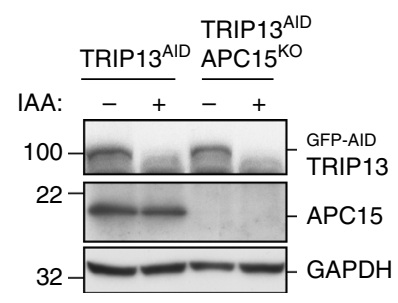

C

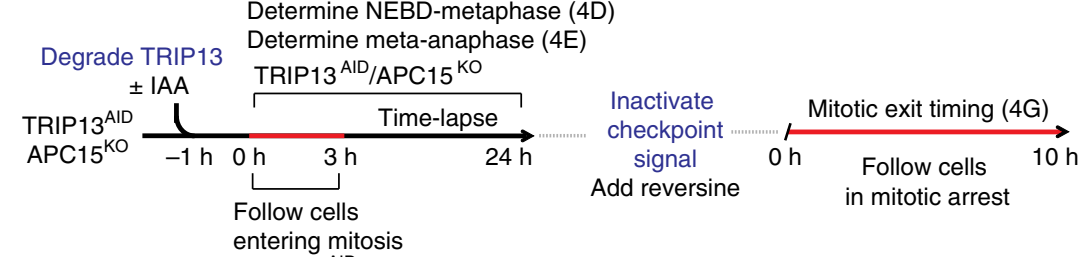

d

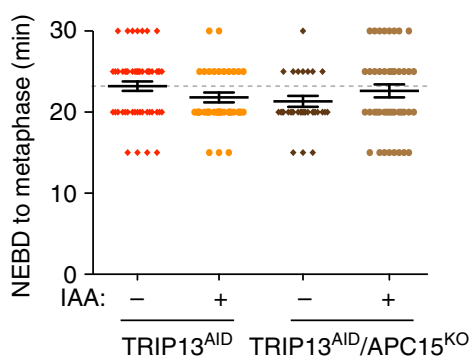

e

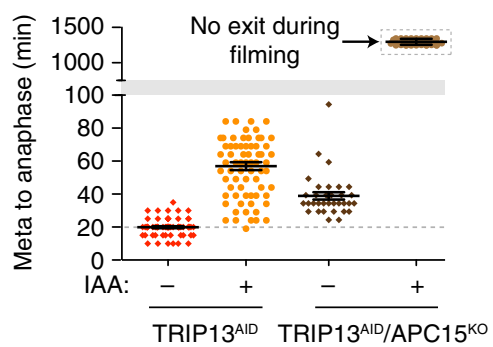

b

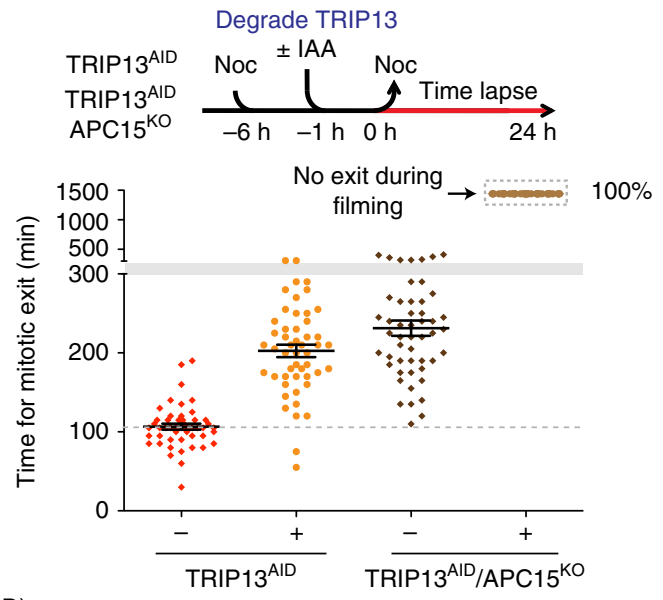

f

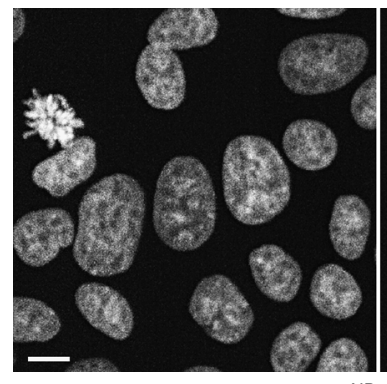

TRIP13 1 AID $/ A P$

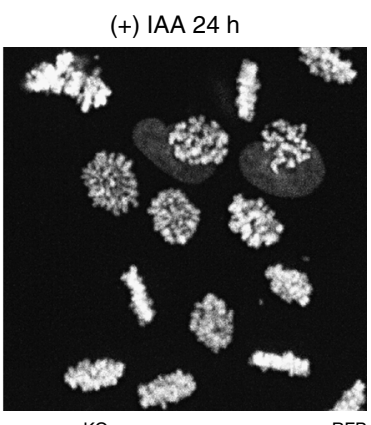

$\mathrm{H}_{2 \mathrm{~B}}^{\mathrm{mRFP}}$ g

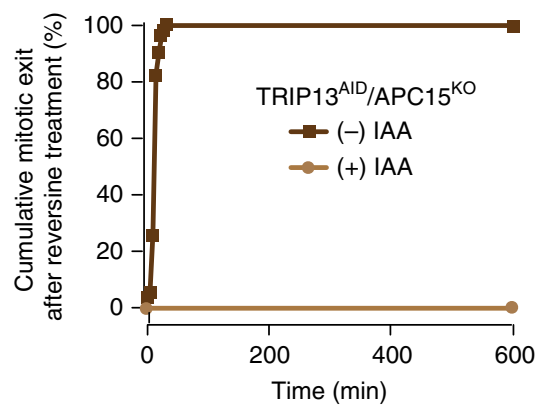

Fig. 3 TRIP13 and APC15 synergistically act on kinetochore-generated MCC. a Western blot showing effective depletion of GFP-AID-TRIP13 in TRIP13AID and TRIP13AID /APC15 ${ }^{\mathrm{KO}}$ cells. b Upper: Schematic of experiment to measure timing of mitotic exit after TRIP13 depletion by IAA addition. Lower: Timing of mitotic exit after 6-h nocodazole treatment, followed by release into DMEM. ( $n=50$ for each experimental condition). c Schematic of approach to monitor unperturbed mitosis after depletion of TRIP13 (by IAA addition) in TRIP13AID/APC15KO cells (used in d-f). d Unperturbed mitotic timing NEBD-tometaphase; ( $n=50$ for each experimental condition). e Metaphase-to-anaphase of TRIP13AID and TRIP13AID/APC15 KO cells entering mitosis $3 \mathrm{~h}$ after depletion of TRIP13. ( $n=70$ for each experimental condition). f Representative images of chromosomes in TRIP13 AID/APC15 ${ }^{\mathrm{KO}}$ cells after before IAA treatment (left) and $24 \mathrm{~h}$ after IAA treatment (right). Scale bar, $5 \mu \mathrm{m}$. $\mathbf{g}$ Mitotic exit timing after inhibition of kinetochore signaling. Reversine was added $24 \mathrm{~h}$ after the start of imaging to inactivate kinetochore signaling, and mitotic exit of cells previously arrested in mitosis was measured for $10 \mathrm{~h}$. $(n=70$ for each experimental condition). Lines in $\mathbf{b}$, $\mathbf{d}$, and $\mathbf{e}$ represent the mean \pm s.e.m.

$\mathrm{kDa} \mathrm{APC} / \mathrm{C}$ subunit that is dispensable for overall $\mathrm{APC} / \mathrm{C}^{\mathrm{Cdc} 20}$ assembly and ubiquitination activity, has been implicated as a required factor for ubiquitination of Cdc20 in MCC-bound $\mathrm{APC} / \mathrm{C}^{\mathrm{Cdc} 20}$ through its promotion of a conformational change in APC/C, which allows recruitment of an E2 enzyme and ubiquitination of Cdc20 in MCC $^{41,42}$.
We tested whether mitotic exit in cells shortly after TRIP13 degradation depended on APC15-mediated Cdc20 ubiquitination and degradation. To do this, we inactivated both APC15 alleles using CRISPR-Cas9 in TRIP13 AID cells (Fig. 3a, hereafter, TRIP13 $\left.{ }^{\mathrm{AID}} / \mathrm{APC} 15^{\mathrm{KO}}\right)$. We treated cells with nocodazole to induce a mitotic checkpoint-mediated arrest, then released from 
nocodazole after rapid depletion of TRIP13 with auxin-induced degradation, and tracked cell-cycle progression by live-cell microscopy. While cells depleted of either APC15 or TRIP13 showed delayed mitotic exit relative to wild-type cells, these cells did eventually exit mitosis (Fig. 3b). In contrast, 100\% of cells depleted of both APC15 and TRIP13 were chronically arrested in mitosis with none escaping over $20 \mathrm{~h}$ of filming (Fig. 3b). We next tracked unperturbed mitosis in TRIP13 ${ }^{\mathrm{AID}}$ / APC $15^{\mathrm{KO}}$ cells, and found that while these cells had normal NEBD-to-metaphase timing and only a slight delay in mitotic exit when TRIP13 was present (Fig. 3d, e), degradation of TRIP13 by IAA treatment produced a durable metaphase arrest, with all cells remaining arrested for the duration of filming (21-24 h) (Fig. 3e, f). Finally, we treated mitotically arrested TRIP13 $3^{\mathrm{AID}} / \mathrm{APC} 15^{\mathrm{KO}}$ cells with reversine to eliminate kinetochore production of MCC. We found that while cells retaining TRIP13 exited mitosis quickly, 100\% of cells lacking both APC15 and TRIP13 (after IAA addition) remained mitotically arrested for at least $10 \mathrm{~h}$ (Fig. 3g). Thus, in the absence of the known catalytic pathways for MCC disassembly or degradation, cells are completely unable to reactivate $\mathrm{APC} / \mathrm{C}^{\mathrm{CdC} 20-\mathrm{MCC}}$ and exit mitosis, even in the absence of any kinetochore signaling.

Mitotic exit relies on turnover of interphase-generated MCC. Recently, multiple studies have indicated that in addition to being assembled at unattached kinetochores in mitosis, MCC may be generated at low levels outside of mitosis, likely by Mad1:Mad2 complexes localized at nuclear pores ${ }^{10,11}$. We tested if this pool of MCC produced in interphase might be enough to delay mitotic exit in the absence of the two catalytic MCC disassembly/degradation pathways. We depleted TRIP13 and simultaneously added reversine to eliminate kinetochore-dependent mitotic checkpoint signaling in TRIP13 $\mathrm{AID} / \mathrm{APC} 15^{\mathrm{KO}}$ cells, and examined mitotic timing. Strikingly, $75 \%$ of cells lacking both APC15 and TRIP13 and which never activated the mitotic checkpoint remained arrested in mitosis for the duration of $24 \mathrm{~h}$ of filming (Fig. 4a). This prolonged mitotic arrest was dependent on both Mad2 and BubR1 (Fig. 4b, Supplementary Fig. 4C), as would be expected from a bona fide MCC-mediated delay. These data not only support the idea that a pool of MCC is synthesized in interphase independently of kinetochores, but also demonstrate that this interphase-produced pool of MCC is sufficient to cause sustained mitotic arrest in the absence of TRIP13 (and its catalytic MCC disassembly activity) or APC15 (and its stimulation of ubiquitination/degradation of the Cdc20 subunit of MCC).

In interphase, Mad1:Mad2 are bound to the nucleoplasmic face of the nuclear envelope through the TPR protein, and this pool of Mad1:Mad2 has been implicated in production of MCC during interphase ${ }^{9,11,23}$. To examine whether TPR (and its tethering of Mad1:Mad2 to the nuclear pore ${ }^{11}$ ) is essential for MCC generation by Mad1:Mad2 at the nuclear pore that is sufficient to trigger sustained mitotic arrest in the absence of TRIP13 and APC15, we used RNAi to deplete TPR from TRIP13AID/ APC15 ${ }^{\mathrm{KO}}$ cells (Supplementary Fig. 4,B) and monitored mitotic timing (Fig. 4c). Like TPR-containing cells (Fig. 4a), TRIP13AID/ APC15 ${ }^{\mathrm{KO}}$ cells entering mitosis after depletion of TPR (but competent for kinetochore-dependent mitotic checkpoint activation) were almost completely (98\%) arrested after IAA-induced degradation of TRIP13 (Fig. 4c). However, when mitotic checkpoint signaling was prevented (by addition of reversine), reduced TPR levels sharply reduced the proportion of mitotically arrested cells [from $75 \%$ (Fig. 4a) to $28 \%$ (Fig. 4c)], consistent with TPR-dependent production of an APC/C $\mathrm{Cd} 220$ inhibitor.

Finally, we tested for the presence of MCC (either free or bound to $\mathrm{APC} / \mathrm{C}^{\mathrm{Cdc} 20}$ ) in interphase TRIP $13^{\mathrm{AID}} / \mathrm{APC} 15^{\mathrm{KO}}$ cells.
Cells were synchronized in either mid-G1 (by inhibiting CDK4/6 with Palbociclib) or in late G2 (by inhibiting CDK1 with RO3306) and the levels of BubR1-bound Cdc20 and Mad2 determined by immunoprecipitation and immunoblotting (Supplementary Fig. 4D). In both G1 and late G2, low levels of APC/ C-associated BubR1 and Mad2 were detected in extracts from cells containing TRIP13; much higher levels were found in extracts of cells depleted of TRIP13 (by IAA-induced degradation) (Fig. 4d). Overall MCC levels (as measured by BubR1bound Mad2) also increased upon degradation of TRIP13 in G2arrested cells (Supplementary Fig. 4D). These data support a role for TRIP13 in continual MCC disassembly throughout interphase as well as mitosis.

To test whether MCC is bound to APC/C during interphase when both TRIP13 and APC15 are missing, we monitored the level of MCC components associated with the core APC/C subunit APC3 in G1 or late G2-arrested TRIP13 $3^{\mathrm{AID}} / \mathrm{APC} 15^{\mathrm{KO}}$ cells. While a small proportion of BubR1 was recruited to APC/C in the presence of TRIP13 in G1 cells (Fig. 4d), cells lacking APC15 and TRIP13 accumulated up to $\sim 8$-fold higher levels of APC/C-bound BubR1, Mad2, and Cdc20 (Fig. 4d). When combined with our evidence for chronic mitotic arrest in the absence of TRIP13, APC15, and mitotic checkpoint activation, these data provide strong evidence that MCC produced in interphase can quantitatively inhibit $\mathrm{APC} / \mathrm{C}^{\mathrm{Cdc} 20}$ and produce chronic mitotic arrest when TRIP13 and APC15 are absent.

\section{Discussion}

Our use of rapid, inducible degradation of TRIP13 has demonstrated that Mad2 conformation in vivo is highly dependent on TRIP13 and its ATPase activity, with cellular O-Mad2 converted to C-Mad2 with a half-time of $1.4 \mathrm{~h}$ in the absence of TRIP13 (Fig. 2a). Since this matches the kinetics of spontaneous conversion of purified O-Mad2 to C-Mad2 in vitro ${ }^{61}$, our evidence offers strong support that cellular O-Mad2 undergoes spontaneous conformational conversion in vivo that is independent of other factors. We also show that after loss of TRIP13, insufficient soluble O-Mad2 at the time of mitotic entry results in failure of mitotic checkpoint activation and that checkpoint activation can be rescued by re-introduction of additional O-Mad2. Together, these observations establish that a key function of TRIP13 throughout the cell cycle is to counteract the spontaneous conversion of O-Mad2 to C-Mad2, thereby maintaining a pool of soluble O-Mad2 which can be recruited to kinetochores in mitosis to produce MCC (Fig. 5) and clarify the dual roles of TRIP13 in mitotic checkpoint activation and silencing.

Our data also provide strong support for the idea that MCC is assembled outside of mitosis by Mad1, Mad2, and TPR, the latter of which tethers Mad1-Mad2 to the nuclear pore complex ${ }^{11,23}$. Our evidence suggests that APC15 and TRIP13 mediate continuous turnover of interphase-produced MCC complexes, which act both to restrain the activity of any $\mathrm{APC} / \mathrm{C}^{\mathrm{Cdc} 20}$ present in interphase, and provide a baseline level of pre-made MCC to delay mitotic exit prior to kinetochore maturation and mitotic checkpoint activation. Thus, TRIP13 and APC15 activity serve as a counter-balance to MCC assembly both in interphase and in mitosis ${ }^{11}$. The delicate balance between MCC assembly and disassembly/degradation pathways, and these pathways' coordination through space and time, will be important to consider as the contributions to cancer of both TRIP13 loss and overexpression are further studied.

Mitotic checkpoint silencing is initiated when spindle microtubules capture all kinetochores, thereby suppressing production of new MCC through delocalization of kinetochore-bound Mad1: C-Mad2 $2^{24-27}$ and reduced activity of kinetochore-bound 
a

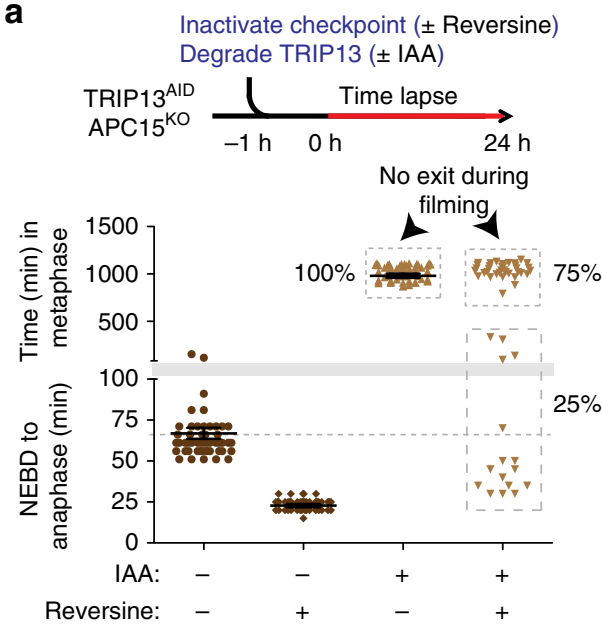

C

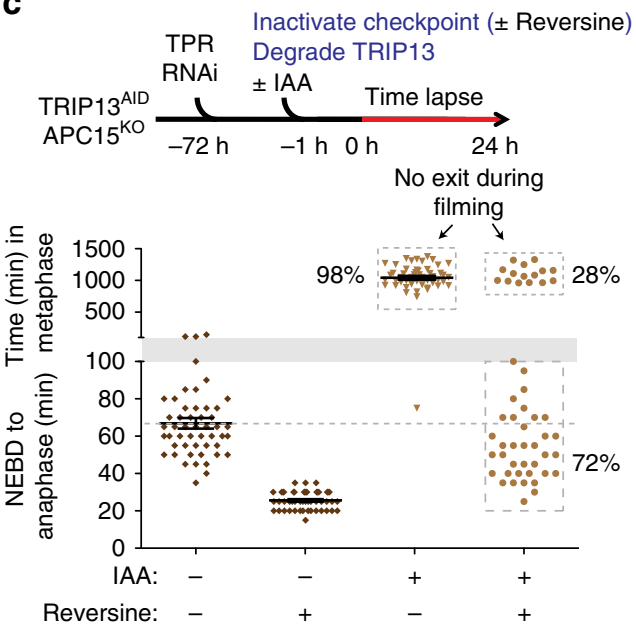

b

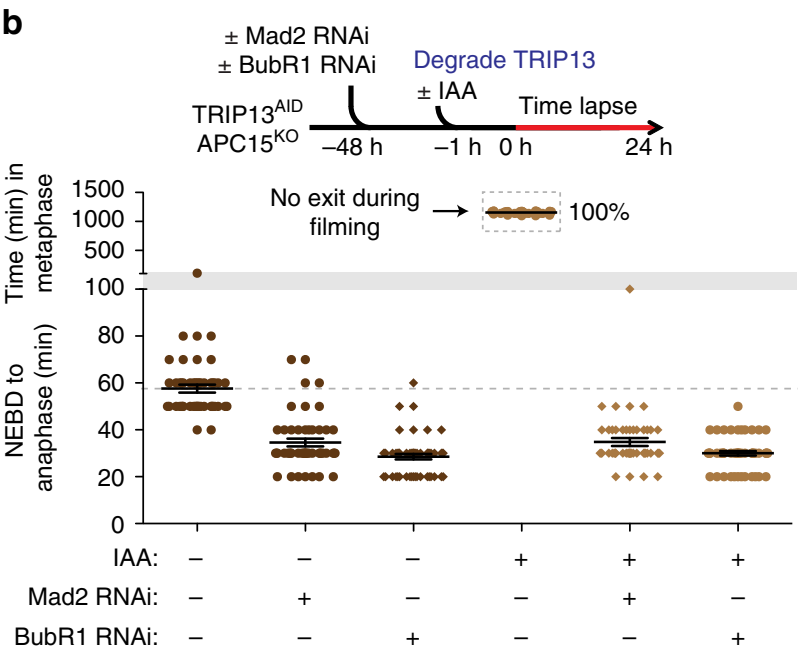

d

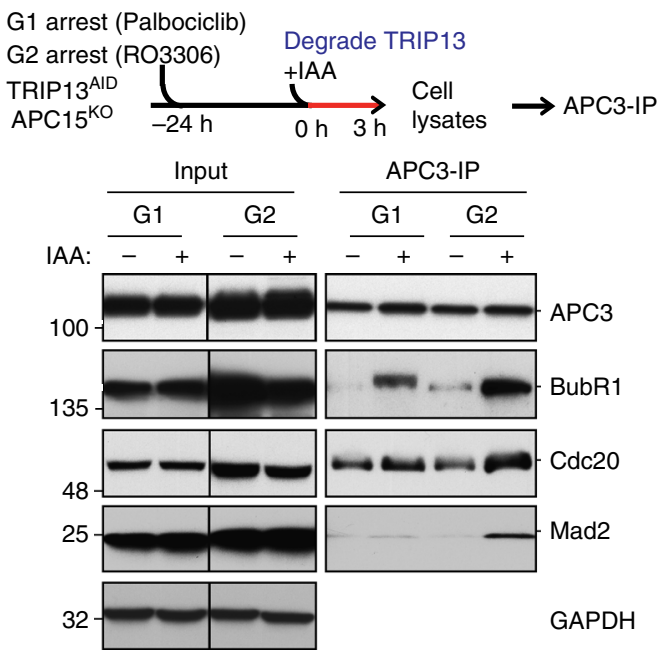

Fig. 4 TRIP13 and APC15 are required for turnover of MCC produced in interphase. a Upper: Schematic of experiment to measure the dependence of the observed mitotic exit delay on kinetochore-catalyzed MCC production. Lower: mitotic timing in reversine-treated TRIP13 ${ }^{A I D} / A P C 15^{K O}$ cells in the presence or absence of IAA. ( $n=50$ for each experimental condition). $\mathbf{b}$ Upper: Schematic of experiment to measure the dependence of the observed mitotic exit delay on Mad2 and BubR1. Lower: mitotic timing in Mad2 or BubR1 RNAi treated TRIP13AID/APC15 KO cells in the presence or absence of IAA. ( $n=50$ for each experimental condition). c Upper: Schematic of experiment to measure the dependence of the observed mitotic exit delay on Tpr. Lower: mitotic timing in Tpr RNAi treated TRIP13AID /APC15 ${ }^{\mathrm{KO}}$ cells in the presence or absence of reversine and IAA. ( $n=50$ for each experimental condition). d Upper: Schematic of experiment to measure APC/CMCC formation in TRIP13AID/APC15 KO. APC3 was immunoprecipitated from cells in G1 with Palbociclib and in late G2 with RO-3306. Lower: APC/C interacting BubR1 and Cdc20 levels were measured by quantitative immunoblotting. Lines in a-c represent the mean \pm s.e.m.

checkpoint kinases ${ }^{28-33}$. A key question has been how pre-existing free and $\mathrm{APC} / \mathrm{C}^{\mathrm{Cdc} 20}$-bound MCC are inactivated by disassembly or degradation so as to reactivate $\mathrm{APC} / \mathrm{C}^{\mathrm{Cdc} 20}$. We show here that two separate catalytic pathways (TRIP13/p31 ${ }^{\text {comet }}$-mediated MCC disassembly through C-Mad2 to O-Mad2 conformational conversion ${ }^{47-50,52}$ and APC15-dependent ubiquitination of Cdc20 in MCC) are essential for inactivating MCC made either in interphase or in mitosis. Indeed, we have found that once assembled, MCC complexes are extraordinarily stable, with a negligible rate of spontaneous inactivation, producing chronic mitotic arrest in cells lacking both MCC disassembly and degradation pathways even in the absence of actively signaling kinetochores (Fig. 5). Recently, Brulotte et al. ${ }^{66}$ showed that in vitro low levels of TRIP13 were unable to quickly reactivate $\mathrm{APC} / \mathrm{C}^{\mathrm{Cdc} 20}$ that had been preincubated with MCC components, but were sufficient to disassemble-free MCC and prevent APC/C $\mathrm{Cdc}^{\mathrm{Cd} 20}$ inhibition. These data clearly show that free MCC is the preferred substrate of
TRIP13, but does not eliminate the possibility of low-level activity on APC/C-MCC complexes. Thus, while our data show that both MCC disassembly pathways on their own are capable of mediating eventual mitotic exit, we postulate that in wild-type cells, a division of labor exists where TRIP13/p31 comet preferentially disassembles free MCC, and APC15 catalyzes disassembly/ degradation of APC/C-bound MCC.

Cellular studies and recent high-resolution structures of MCCbound $\mathrm{APC} / \mathrm{C}^{\mathrm{Cdc} 20}$ have revealed that multiple degron sequences in the BubR1 subunit of MCC occupy the substrate-binding sites of $\mathrm{Cdc} 20$ to inhibit APC/C activity ${ }^{41,42}$. It is striking, then, that the two MCC degradation/disassembly pathways do not target BubR1 directly, instead targeting either Mad2 (in the case of TRIP13/p31 ${ }^{\text {comet}) ~ o r ~ C d c 20 ~(i n ~ t h e ~ c a s e ~ o f ~ A P C 15-m e d i a t e d ~}$ ubiquitination). We have shown that while cells normally employ both pathways in parallel, either pathway is sufficient for eventual mitotic exit. These data (1) reveal that both pathways can act on APC/C $\mathrm{C}^{\mathrm{Cdc} 20}$-bound MCC (TRIP13 can also likely act on soluble 


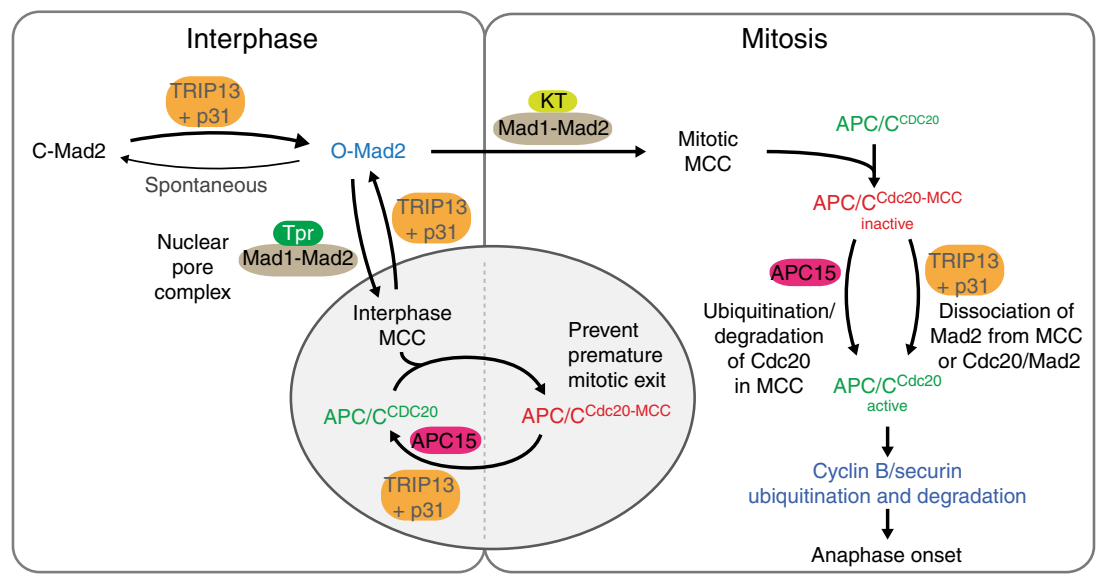

Fig. 5 Model for TRIP13 and APC15 contributions to mitotic checkpoint signaling. In interphase cells, TRIP13 and p31 comet counteract the spontaneous conversion of O-Mad2 to C-Mad2 in order to maintain a pool of O-Mad2 for checkpoint activation and regulate the homeostasis of both Mad2 and p31 $1^{\text {comet. }}$. Additionally, TRIP13 and APC15 together prevent formation of excess MCC during interphase for proper mitotic exit in the next mitosis. In prometaphase, conversion of O-Mad2 to Cdc2O-bound C-Mad2 at unattached kinetochores catalyzes assembly of MCC, which binds and inhibits $\mathrm{APC} / \mathrm{C}^{\mathrm{Cdc} 20}$. Checkpoint silencing at metaphase can occur through two pathways: First, TRIP13 and p31comet can catalyze the disassembly of free MCC and the removal/disassembly of $\mathrm{MCC}$ bound to APC/CCdc20. Second, APC15-mediated conformational changes within the APC/C can allow ubiquitination of $\mathrm{Cdc} 20$ in $\mathrm{MCC}$, followed by reactivation of $\mathrm{APC} / \mathrm{C}^{\mathrm{Cdc} 20}$

MCC) and (2) show that disruption of MCC structure by removal of either Mad2 or Cdc20 is sufficient to relieve inhibition of APC/ $\mathrm{C}^{\mathrm{Cdc} 20}$ from ubiquitination of cyclin $\mathrm{B}$ and securin. Earlier evidence $^{67}$ that nocodazole-treated cells depleted of TRIP13 activity (by reduction of its $\mathrm{p} 31^{\text {comet }}$ adapter) remain mitotically arrested after induced degradation of Mad2, but not BubR1, suggests that TRIP13's action on APC/C Cdc20-MCC may remove BubR1 along with Mad2 or destabilize the remaining MCC complex ${ }^{22}$. Indeed, BubR1 release may lag behind removal of Mad2-potentially explaining earlier observations of $\mathrm{APC} / \mathrm{C}^{\mathrm{Cdc} 20}$-bound "BBC" complex (BubR1, Bub3, Cdc20) ${ }^{21,43,68}$, but it nonetheless eventually dissociates to enable $\mathrm{APC} / \mathrm{C}^{\mathrm{Cdc} 20}$ reactivation.

\section{Methods}

DNA constructs and site-directed mutagenesis. Full-length ORFs of TRIP13 were obtained from OriGene technologies (Rockville, MD). cDNAs of TRIP13 were cloned into a pcDNA5/FRT/TO-based vector (Invitrogen) modified to contain an amino-terminus LAP tag. pCDNA5/FRT/TO-Mad2WT was generated as described in previous study ${ }^{52}$. TRIP13 ${ }^{\mathrm{EQ}}$ variant (E253Q) was generated by QuickChangeII site-directed mutagenesis kit (Agilent).

Cell culture and generation of stable cell lines. All the cell lines used in this study were cultured in Dulbecco's Modified Eagle's Medium (DMEM, Thermo Fisher) supplemented with $10 \%$ tetracycline-free fetal bovine serum (Omega Scientific) and $100 \mathrm{U} / \mathrm{mL}$ penicillin-streptomycin and maintained at $37^{\circ} \mathrm{C}$ under $5 \%$ $\mathrm{CO}_{2}$. TRIP13 ${ }^{\mathrm{AID}}$ cells; TIR1 and H2B ${ }^{\mathrm{mRFP}}$-expressing T-REx Flp-In DLD-1 cells ${ }^{60}$ were engineered by CRISPR/Cas 9 and HR-mediated genome editing to integrate green fluorescent protein (GFP) and auxin-inducible degron (AID) in front of TRIP13 locus (AID ORF was a kind gift from Masato Kanemaki; National Institute of Genetics, Mishima, Japan).

Oligonucleotides containing sgRNA sequences targeting TRIP13 are inserted into BbsI site in pX330-U6-Chimeric_BB-CBh-hSpCas9 (Addgene 42230). pCR4Blunt TOPO (Invitrogen) containing EGFP-AID cDNA with two TRIP13

homology arms ( $750 \mathrm{bp}$, respectively) at both ends was constructed using Gibson assembly method (NEB). Two plasmids were co-transfected into DLD-1 TRex FlpIn TIR1-9Myc via electroporation. Two days after transfection, GFP-positive single cells were isolated using single-cell sorting (FACS-Vantage; Becton Dickinson). Targeted cells were identified through genomic PCR and DNA sequencing, further confirmed by immunoblotting. To generate TRIP13 ${ }^{\mathrm{AID}} / \mathrm{APC} 15^{\mathrm{KO}}$ cells, C11orf51 CRISPR/Cas9 KO Plasmid (Santa Cruz Biotechnology) was transfected into TRIP13 ${ }^{\text {AID }}$ cells and targeted cells were identified by immunoblotting.

For generation stable cell line inducibly expressing LAP-TRIP13 WT/EQ and Mad2 WT-Flp, pcDNA5/FRT/TO-LAP-TRIP13 or Mad2 were co-transfected with pOG44 into TRIP13 ${ }^{\text {AID }}$ cells using X-tremeGENE 9 (Roche). Cells were selected with $150 \mu \mathrm{g} / \mathrm{mL}$ hygromycin B (Thermo Fisher), and protein expression was confirmed by immunoblotting.
Doxycycline and the indole-3-acetic acid (IAA) were dissolved in water and used at $1 \mu \mathrm{g} / \mathrm{mL}$ and $500 \mu \mathrm{M}$, respectively. For mitotic arrest, $0.33 \mu \mathrm{M}$ nocodazole (Sigma Aldrich) was used. For checkpoint inactivation, $2 \mu \mathrm{M}$ reversine (Santa Cruz Biotechnology) was used.

RNA interference. For depletion of endogenous proteins, TRIP13 ${ }^{\mathrm{AID}}$ cells were transfected with $50 \mathrm{nM}$ of siRNA and Lipofectamine RNAiMAX (Thermo Fisher) for $48 \mathrm{~h}$ (remaining) before analysis. siRNAs directed against the $3^{\prime}$ untranslated region of Mad2, BubR1 or On-TARGETplus-non targeting siRNA; were purchased from GE Dharmacon.

Time-lapse live-cell imaging. To determine mitotic timing, TRIP13 ${ }^{\text {AID }}$ cells were seeded onto CELLSTAR $\mu$ Clear 96-well plate (Greiner bio-one) and images were collected using a CQ1 confocal image cytometer (Yokogawa Electric Corporation) with a $\times 40$ objective. $5 \times 2$ - $\mu \mathrm{m} z$-sections in RFP $(20 \%$ power, $150 \mathrm{~ms}, 30 \%$ gain) were acquired in each field at $2.5,5$, or 10 -min intervals. Image acquisition and data analysis were performed using ImageJ. To measure mitotic error rate, chromosome lagging and chromosome misalignment were counted during anaphase onset.

Cell growth, flow cytometry, and clonogenic assays. For cell doubling time measurements, cells were plated into six-well dishes in triplicate and counted at three days intervals. For flow cytometry analysis, ethanol-fixed cells were stained with $1 \mu \mathrm{g} / \mathrm{mL}$ Hoechst 33258 (Thermo Fisher) and Phospho Histone H3 (Ser10) antibody conjugated with Alexa647 (Cell Signaling) and analyzed with a SH800 cell sorter (Sony). For confirmation of cell-cycle synchronization, ethanol-fixed cells were stained with $10 \mu \mathrm{g} / \mathrm{mL}$ propidium iodide and $50 \mu \mathrm{g} / \mathrm{mL}$ RNase A and analyzed for DNA content by flow cytometry on a BD LSR II instrument (BD Biosciences). For clonogenic growth assays, 100 cells were plated into six-well dishes in triplicate for 21 days. Methanol-fixed colonies were stained with a $0.5 \%$ crystal violet, $25 \%$ methanol solution, and quantified using ImageJ software.

DNA fluorescent in situ hybridization (FISH). For FISH, TRIP13 ${ }^{\mathrm{AID}}$ cells were cultured in chambered slides (IBIDI) with or without IAA for 5 days and fixed in cold methanol:acetic acid (3:1) for 15 mins and dehydrated with $80 \%$ ethanol.

Centromere 4 enumeration painting probes (MetaSystems) was applied to slides, sealed with a coverslip, co-denatured at $75^{\circ} \mathrm{C}$ for $2 \mathrm{mins}$, and hybridized overnight at $37^{\circ} \mathrm{C}$ in a humidified chamber. Slides were subsequently washed with $0.4 \times$ SCC at $72{ }^{\circ} \mathrm{C}$ for 2 mins and rinsed in $2 \times$ SCC, $0.05 \%$ Tween- 20 at room temperature for $30 \mathrm{~s}$. Slides were then rinsed in water, counterstained with DAPI, and mounted in anti-fade solution. FISH images were acquired on a DeltaVision Core system (GE Health Sciences) at $\times 60$ magnification $(5 \times 1 \mu \mathrm{m} z$-sections) and maximum intensity projections were generated using softWoRx software.

Ion exchange and size-exclusion chromatography. For Mad 2 conformation and complexes formation analyses, we prepared fresh whole-cell lysate without freeze and thaw to prevent conformation changes during preparation. For examination of Mad2 conformation, ion-exchange chromatography was carried out as described in Ye et al. ${ }^{52}$ Whole-cell lysates were injected onto a Mono-Q column (GE 
Healthcare) in buffer containing $25 \mathrm{mM}$ Tris pH 7.4, $1 \mathrm{mM} \mathrm{MgCl} 2,1 \mathrm{mM}$ EDTA, 1 $\mathrm{mM}$ DTT and $50 \mathrm{mM} \mathrm{NaCl}$, and then eluted with a gradient to $400 \mathrm{mM} \mathrm{NaCl}$. For examination of Mad2 complex formation, whole-cell lysates were injected onto a Superdex 200 column (GE Healthcare). Fractions were collected and analyzed by SDS-PAGE followed by immunoblot against Mad2, p31 ${ }^{\text {comet }}$, and Mad1 antibodies.

Antibodies and immunoprecipitation. BubR1 $1^{1-322}$ was expressed in protein was expressed in E. coli Rosetta2 (DE3) pLysS by induction with IPTG for $20 \mathrm{~h}$ at $20^{\circ} \mathrm{C}$, then purified by $\mathrm{Ni}^{2+}$ affinity (Ni-NTA; Qiagen). The protein was used to immunize a rabbit for antisera. Anti-BubR1 antibody was purified by antigen crosslinked HiTrap NHS-activated HP (GE Healthcare) column. Rabbit antiBubR1 (homemade, 1:10000), rabbit anti-TRIP13 (Bethyl, Cat\# A303-605, 1:3000), rabbit anti-Mad2 (Bethyl, Cat\# A300-301A, 1:3000), mouse anti-p31 $1^{\text {comet }}$

(Mad2L1BP) (Santa Cruz, Cat\# SC-134381, 1:200), mouse anti-Cdc20 (Santa Cruz, Cat\# SC-136024, 1:200), mouse anti-APC3 (Cdc27) (Santa Cruz, Cat\# SC-9972, 1:200), mouse anti-GAPDH (6C5) (Abcam, AB8245, 1:10000), and mouse anti- $\alpha$ tubulin (DM1 $\alpha$ ) (Abcam, AB7291, 1:10000) were used for immunoblotting. To monitor $\mathrm{MCC}$ and $\mathrm{APC} / \mathrm{C}^{\mathrm{MCC}}$ formation, rabbit anti-BUBR1 or mouse anti-APC3 were conjugated to M-270 epoxy Dynal bead using Dynabead antibody coupling kit (Thermo Fisher). For immunoprecipitation, conjugated antibodies were mixed with precleared whole-cell lysates that have $1 \mathrm{mg}$ total protein, and rotated for 90 min at $4{ }^{\circ} \mathrm{C}$. After three times washing with lysis buffer $(20 \mathrm{mM}$ Tris- $\mathrm{HCl}, \mathrm{pH} 7.4$, $150 \mathrm{mM} \mathrm{NaCl}, 1 \mathrm{mM}$ EDTA, $0.2 \% \mathrm{NP}-40$ ), the beads were treated with $30 \mu \mathrm{L} 2 \times$ SDS sample buffer and boiled for $5 \mathrm{~min}$. Uncropped western blots are shown in Supplementary Figure 5.

Protein purification. For in vitro characterization of wild-type Mad2 or $\mathrm{Mad} 2{ }^{\mathrm{H} 191 \mathrm{~A}}$ (both of which contained the R133A mutation to reduce homodimerization), protein was expressed in E. coli Rosetta2 (DE3) pLysS by induction with IPTG for $20 \mathrm{~h}$ at $20^{\circ} \mathrm{C}$, then purified by $\mathrm{Ni}^{2+}$ affinity (Ni-NTA; Qiagen) and ion-exchange (HiTrap Q HP; GE Life Sciences) chromatography. His $_{6}{ }_{6}$-SUMO tags were cleaved by incubation with $S$. cerevisiae Ulp 1 at $4{ }^{\circ} \mathrm{C}$ overnight, and cleaved protein was passed over a $\mathrm{Ni}^{2+}$ affinity column a second time to remove uncleaved protein, $\mathrm{His}_{6}$-SUMO tags, and Ulp1. Protein was purified by size-exclusion chromatography (Superdex 200, GE Life Sciences), concentrated by ultrafiltration, and stored at $-80^{\circ} \mathrm{C}$ for biochemical assays.

Quantification and statistical analysis. For quantification of TRIP13 and Mad2, triplicate samples were analyzed on independent Western blots, band intensity measured by ImageJ, and points plotted as mean \pm standard deviation. Degradation/conformational conversion half-times were calculated in Prism v. 7 using a single-exponential decay model (plateau value set to $0 \%$ for TRIP13 degradation, but allowed to float for MAD2 conversion). For analysis of cell-cycle timing, at least 50 cells were tracked by live-cell imaging at 1-5 min intervals (depending on experiment), and are plotted as mean \pm s.e.m. (standard error of the mean). For all panels showing $P$-values, these were calculated in Prism v. 7 using an unpaired two-tailed $t$-test $\left({ }^{*} P<0.01,{ }^{* *} P<0.001, \mathrm{NS}=\right.$ not significant).

\section{Data availability}

The data that support the findings of this study are available from the authors on reasonable request, see author contributions for specific data sets.

Received: 10 May 2018 Accepted: 13 September 2018

Published online: 19 October 2018

\section{References}

1. Irniger, S., Piatti, S., Michaelis, C. \& Nasmyth, K. Genes involved in sister chromatid separation are needed for B-type cyclin proteolysis in budding yeast. Cell 81, 269-278 (1995).

2. Sudakin, V. et al. The cyclosome, a large complex containing cyclin-selective ubiquitin ligase activity, targets cyclins for destruction at the end of mitosis. Mol. Biol. Cell 6, 185-197 (1995).

3. King, R. W. et al. A $20 \mathrm{~S}$ complex containing CDC27 and CDC16 catalyzes the mitosis-specific conjugation of ubiquitin to cyclin B. Cell 81, 279-288 (1995).

4. Tugendreich, S., Tomkiel, J., Earnshaw, W. \& Hieter, P. CDC27Hs colocalizes with $\mathrm{CDC} 16 \mathrm{Hs}$ to the centrosome and mitotic spindle and is essential for the metaphase to anaphase transition. Cell 81, 261-268 (1995).

5. Pines, J. Cubism and the cell cycle: the many faces of the APC/C. Nat. Rev. Mol. Cell Biol. 12, 427-438 (2011).

6. Li, Y., Gorbea, C., Mahaffey, D., Rechsteiner, M. \& Benezra, R. MAD2 associates with the cyclosome/anaphase-promoting complex and inhibits its activity. Proc. Natl Acad. Sci. USA 94, 12431-12436 (1997).

7. Fang, G., Yu, H. \& Kirschner, M. W. The checkpoint protein MAD2 and the mitotic regulator CDC20 form a ternary complex with the anaphase- promoting complex to control anaphase initiation. Genes Dev. 12, 1871-1883 (1998).

8. Sudakin, V., Chan, G. K. \& Yen, T. J. Checkpoint inhibition of the APC/C in HeLa cells is mediated by a complex of BUBR1, BUB3, CDC20, and MAD2. J. Cell Biol. 154, 925-936 (2001).

9. Schweizer, N. et al. Spindle assembly checkpoint robustness requires Tprmediated regulation of Mad1/Mad2 proteostasis. J. Cell Biol. 203, 883-893 (2013).

10. Meraldi, P., Draviam, V. M. \& Sorger, P. K. Timing and checkpoints in the regulation of mitotic progression. Dev. Cell 7, 45-60 (2004).

11. Rodriguez-Bravo, V. et al. Nuclear pores protect genome integrity by assembling a premitotic and Mad1-dependent anaphase inhibitor. Cell 156, 1017-1031 (2014).

12. Tipton, A. R. et al. BUBR1 and closed MAD2 (C-MAD2) interact directly to assemble a functional mitotic checkpoint complex. J. Biol. Chem. 286, 21173-21179 (2011)

13. Aravind, L. \& Koonin, E. V. The HORMA domain: a common structural denominator in mitotic checkpoints, chromosome synapsis and DNA repair. Trends Biochem. Sci. 23, 284-286 (1998).

14. Luo, X., Tang, Z., Rizo, J. \& Yu, H. The Mad2 spindle checkpoint protein undergoes similar major conformational changes upon binding to either Mad1 or Cdc20. Mol. Cell 9, 59-71 (2002).

15. Sironi, L. et al. Crystal structure of the tetrameric Mad1-Mad2 core complex: implications of a 'safety belt' binding mechanism for the spindle checkpoint. EMBO J. 21, 2496-2506 (2002)

16. Mapelli, M., Massimiliano, L., Santaguida, S. \& Musacchio, A. The Mad2 conformational dimer: structure and implications for the spindle assembly checkpoint. Cell 131, 730-743 (2007).

17. Luo, X. et al. The Mad2 spindle checkpoint protein has two distinct natively folded states. Nat. Struct. Mol. Biol. 11, 338-345 (2004).

18. Shah, J. et al. Dynamics of centromere and kinetochore proteinsimplications for checkpoint signaling and silencing. Curr. Biol. 14, 942-952 (2004).

19. Vink, M. et al. In vitro FRAP identifies the minimal requirements for Mad2 kinetochore dynamics. Curr. Biol. 16, 755-766 (2006).

20. Ji, Z., Gao, H., Jia, L., Li, B. \& Yu, H. A sequential multi-target Mps1 phosphorylation cascade promotes spindle checkpoint signaling. eLife $\mathbf{6}$, e22513 (2017).

21. Kulukian, A., Han, J. S. \& Cleveland, D. W. Unattached kinetochores catalyze production of an anaphase inhibitor that requires a Mad2 template to prime Cdc20 for BubR1 binding. Dev. Cell 16, 105-117 (2009).

22. Faesen, A. C. et al. Basis of catalytic assembly of the mitotic checkpoint complex. Nature 542, 498-502 (2017).

23. Lee, S. H., Sterling, H., Burlingame, A. \& McCormick, F. Tpr directly binds to Mad1 and Mad2 and is important for the Mad1-Mad2-mediated mitotic spindle checkpoint. Genes Dev. 22, 2926-2931 (2008)

24. Howell, B. J. et al. Cytoplasmic dynein/dynactin drives kinetochore protein transport to the spindle poles and has a role in mitotic spindle checkpoint inactivation. J. Cell Biol. 155, 1159-1172 (2001).

25. Barisic, M. \& Geley, S. Spindly switch controls anaphase: spindly and RZZ functions in chromosome attachment and mitotic checkpoint control. Cell Cycle 10, 449-456 (2011).

26. Gassmann, R. et al. A new mechanism controlling kinetochore-microtubule interactions revealed by comparison of two dynein-targeting components: SPDL-1 and the Rod/Zwilch/Zw10 complex. Genes Dev. 22, 2385-2399 (2008).

27. Gassmann, R. et al. Removal of Spindly from microtubule-attached kinetochores controls spindle checkpoint silencing in human cells. Genes Dev. 24, 957-971 (2010).

28. Wei, R., Ngo, B., Wu, G. \& Lee, W. H. Phosphorylation of the Ndc80 complex protein, HEC1, by Nek2 kinase modulates chromosome alignment and signaling of the spindle assembly checkpoint. Mol. Biol. Cell 22, 3584-3594 (2011).

29. Zhang, G., Lischetti, T. \& Nilsson, J. A minimal number of MELT repeats supports all the functions of KNL1 in chromosome segregation. J. Cell Sci. 127, 871-884 (2014).

30. Rosenberg, J. S., Cross, F. R. \& Funabiki, H. KNL1/Spc105 recruits PP1 to silence the spindle assembly checkpoint. Curr. Biol. 21, 942-947 (2011).

31. Meadows, J. C. et al. Spindle checkpoint silencing requires association of PP1 to both Spc7 and kinesin-8 motors. Dev. Cell 20, 739-750 (2011).

32. Vanoosthuyse, V. \& Hardwick, K. G. A novel protein phosphatase 1dependent spindle checkpoint silencing mechanism. Curr. Biol. 19, 1176-1181 (2009).

33. Pinsky, B. A., Nelson, C. R. \& Biggins, S. Protein phosphatase 1 regulates exit from the spindle checkpoint in budding yeast. Curr. Biol. 19, 1182-1187 (2009).

34. Pan, J. \& Chen, R. H. Spindle checkpoint regulates Cdc20p stability in Saccharomyces cerevisiae. Genes Dev. 18, 1439-1451 (2004). 
35. Reddy, S. K., Rape, M., Margansky, W. A. \& Kirschner, M. W. Ubiquitination by the anaphase-promoting complex drives spindle checkpoint inactivation. Nature 446, 921-925 (2007).

36. Ge, S., Skaar, J. R. \& Pagano, M. APC/C- and Mad2-mediated degradation of Cdc20 during spindle checkpoint activation. Cell Cycle 8, 167-171 (2009).

37. Foe, I. T. et al. Ubiquitination of Cdc20 by the APC occurs through an intramolecular mechanism. Curr. Biol. 21, 1870-1877 (2011)

38. Mansfeld, J., Collin, P., Collins, M. O., Choudhary, J. S. \& Pines, J. APC15 drives the turnover of MCC-CDC20 to make the spindle assembly checkpoint responsive to kinetochore attachment. Nat. Cell Biol. 13, 1234-1243 (2011).

39. Foster, S. A. \& Morgan, D. O. The APC/C subunit Mnd2/Apc15 promotes $\mathrm{Cdc} 20$ autoubiquitination and spindle assembly checkpoint inactivation. Mol. Cell 47, 921-932 (2012).

40. Uzunova, K. et al. APC15 mediates CDC20 autoubiquitylation by APC/C (MCC) and disassembly of the mitotic checkpoint complex. Nat. Struct. Mol. Biol. 19, 1116-1123 (2012).

41. Alfieri, C. et al. Molecular basis of APC/C regulation by the spindle assembly checkpoint. Nature 536, 431-436 (2016).

42. Yamaguchi, M. et al. Cryo-EM of mitotic checkpoint complex-bound APC/C reveals reciprocal and conformational regulation of ubiquitin ligation. Mol. Cell 63, 593-607 (2016).

43. Westhorpe, F. G., Tighe, A., Lara-Gonzalez, P. \& Taylor, S. S. p31cometmediated extraction of Mad2 from the MCC promotes efficient mitotic exit. J. Cell Sci. 124, 3905-3916 (2011).

44. Yang, M. et al. p31comet blocks Mad2 activation through structural mimicry. Cell 131, 744-755 (2007)

45. Chao, W. C., Kulkarni, K., Zhang, Z., Kong, E. H. \& Barford, D. Structure of the mitotic checkpoint complex. Nature 484, 208-213 (2012).

46. Xia, G. et al. Conformation-specific binding of p31(comet) antagonizes the function of Mad2 in the spindle checkpoint. EMBO J. 23, 3133-3143 (2004).

47. Miniowitz-Shemtov, S., Eytan, E., Kaisari, S., Sitry-Shevah, D. \& Hershko, A. Mode of interaction of TRIP13 AAA-ATPase with the Mad2-binding protein p31comet and with mitotic checkpoint complexes. Proc. Natl Acad. Sci. USA 112, 11536-11540 (2015).

48. Eytan, E. et al. Disassembly of mitotic checkpoint complexes by the joint action of the AAA-ATPase TRIP13 and p31(comet). Proc. Natl Acad. Sci. USA 111, 12019-12024 (2014)

49. Wang, K. et al. Thyroid hormone receptor interacting protein 13 (TRIP13) AAA-ATPase is a novel mitotic checkpoint-silencing protein. J. Biol. Chem. 289, 23928-23937 (2014).

50. Ye, Q. et al. TRIP13 is a protein-remodeling AAA+ATPase that catalyzes MAD2 conformation switching. eLife 4, e07367 (2015)

51. Alfieri, C., Chang, L. \& Barford, D. Mechanism for remodelling of the cell cycle checkpoint protein MAD2 by the ATPase TRIP13. Nature 559, 274-278 (2018).

52. Ye, Q. et al. The AAA + ATPase TRIP13 remodels HORMA domains through $\mathrm{N}$-terminal engagement and unfolding. EMBO J. 36, 2419-2434 (2017).

53. Nelson, C. R., Hwang, T., Chen, P. H. \& Bhalla, N. TRIP13PCH-2 promotes $\mathrm{Mad} 2$ localization to unattached kinetochores in the spindle checkpoint response. J. Cell. Biol. 211, 503-516 (2015).

54. Ma, H. T. \& Poon, R. Y. C. TRIP13 regulates both the activation and inactivation of the spindle-assembly checkpoint. Cell Rep. 14, 1086-1099 (2016).

55. Yost, S. et al. Biallelic TRIP13 mutations predispose to Wilms tumor and chromosome missegregation. Nat. Genet. 49, 1148-1151 (2017).

56. Marks, D. H. et al. Mad2 overexpression uncovers a critical role for TRIP13 in mitotic exit. Cell Rep. 19, 1832-1845 (2017).

57. Larkin, S. E. et al. Identification of markers of prostate cancer progression using candidate gene expression. Br. J. Cancer 106, 157-165 (2012).

58. Banerjee, R. et al. TRIP13 promotes error-prone nonhomologous end joining and induces chemoresistance in head and neck cancer. Nat. Commun. 5, 4527 (2014).

59. Nishimura, K., Fukagawa, T., Takisawa, H., Kakimoto, T. \& Kanemaki, M. An auxin-based degron system for the rapid depletion of proteins in nonplant cells. Nat. Methods 6, 917-922 (2009).

60. Holland, A. J., Fachinetti, D., Han, J. S. \& Cleveland, D. W. Inducible, reversible system for the rapid and complete degradation of proteins in mammalian cells. Proc. Natl Acad. Sci. USA 109, E3350-E3357 (2012).

61. Hara, M., Ozkan, E., Sun, H., Yu, H. \& Luo, X. Structure of an intermediate conformer of the spindle checkpoint protein Mad2. Proc. Natl Acad. Sci. USA 112, 11252-11257 (2015).
62. Fry, D. W. et al. Specific inhibition of cyclin-dependent kinase 4/6 by PD 0332991 and associated antitumor activity in human tumor xenografts. Mol. Cancer Ther. 3, 1427-1438 (2004).

63. Yang, M. et al. Insights into mad2 regulation in the spindle checkpoint revealed by the crystal structure of the symmetric mad2 dimer. PLoS Biol. 6, e50 (2008).

64. Kaisari, S., Sitry-Shevah, D., Miniowitz-Shemtov, S. \& Hershko, A. Intermediates in the assembly of mitotic checkpoint complexes and their role in the regulation of the anaphase-promoting complex. Proc. Natl Acad. Sci. USA 113, 966-971 (2016).

65. Santaguida, S., Tighe, A., D’Alise, A. M., Taylor, S. S. \& Musacchio, A. Dissecting the role of MPS1 in chromosome biorientation and the spindle checkpoint through the small molecule inhibitor reversine. J. Cell Biol. 190, 73-87 (2010).

66. Brulotte, M. L. et al. Mechanistic insight into TRIP13-catalyzed Mad2 structural transition and spindle checkpoint silencing. Nat. Commun. 8 , 1956 (2017).

67. Han, J. S. et al. Catalytic assembly of the mitotic checkpoint inhibitor BubR1Cdc20 by a Mad2-induced functional switch in Cdc20. Mol. Cell 51, 92-104 (2013).

68. Nilsson, J., Yekezare, M., Minshull, J. \& Pines, J. The APC/C maintains the spindle assembly checkpoint by targeting Cdc20 for destruction. Nat. Cell Biol. 10, 1411-1420 (2008).

\section{Acknowledgements}

We thank Andrew Shiau and David Jenkins for sharing resources and their assistance with imaging, Arshad Desai, Pablo Lara-Gonzalez, Nam Hee Kim, members of the Cleveland and Corbett labs for helpful discussions, and acknowledge the Ludwig Institute for Cancer Research (to K.D.C. and D.W.C.), NIH R01 GM104141 (to K.D.C.), NIH K99 CA218871 (to P.L.), IBS-R022-D1-2017-a00 (to J.S.H. and K.M.), NIH R01 GM29513 (to D.W.C.), and R35 GM122476 (to D.W.C.) for funding.

\section{Author contributions}

D.H.K., K.D.C. and D.W.C. designed the experiments. J.S.H., K.M. and M.M. generated TRIP13 ${ }^{\mathrm{AID}}$ cells, D.H.K. generated TRIP13 ${ }^{\mathrm{AID}} / \mathrm{APC} 15^{\mathrm{KO}}$ cells and performed all live-cell experiments, FACS analyses, quantitative immunoblotting, and data analysis. J.S.H. generated BubR1 antibody, P.L. performed FISH with data interpretation and Q.Y. performed Mono-Q ion-exchange chromatography. D.H.K., K.D.C. and D.W.C. wrote the manuscript.

\section{Additional information}

Supplementary Information accompanies this paper at https://doi.org/10.1038/s41467018-06774-1.

Competing interests: The authors declare no competing interests.

Reprints and permission information is available online at http://npg.nature.com/ reprintsandpermissions/

Publisher's note: Springer Nature remains neutral with regard to jurisdictional claims in published maps and institutional affiliations.

\footnotetext{
Open Access This article is licensed under a Creative Commons BY Attribution 4.0 International License, which permits use, sharing,
adaptation, distribution and reproduction in any medium or format, as long as you give appropriate credit to the original author(s) and the source, provide a link to the Creative Commons license, and indicate if changes were made. The images or other third party material in this article are included in the article's Creative Commons license, unless indicated otherwise in a credit line to the material. If material is not included in the article's Creative Commons license and your intended use is not permitted by statutory regulation or exceeds the permitted use, you will need to obtain permission directly from the copyright holder. To view a copy of this license, visit http://creativecommons.org/ licenses/by/4.0/.
}

(c) The Author(s) 2018 